УДК 616.716.4-089.87-089.844

DOI: $10.26435 /$ UC.V0I3(36).505

\author{
И.В. Чайковская ${ }^{1}$, М.Ю. Павленко ${ }^{1}$, С.Н. Царенко ${ }^{2}$ \\ ${ }^{1}$ ГОО ВПО «Донецкий национальный медицинский университет имени М. Горького», Донецк

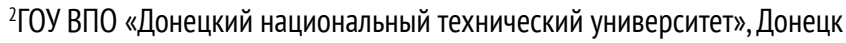

\title{
КОНЕЧНО-ЭЛЕМЕНТАРНЫЙ АНАЛИЗ НАПРЯЖЕННО-ДЕФОРМИРОВАННОГО СОСТОЯНИЯ СТАНДАРТНОЙ И ИНДИВИДУАЛЬНОЙ РЕКОНСТРУКТИВНОЙ ПЛАСТИН ДЛЯ ЗАМЕЩЕНИЯ ДЕФЕКТА ВЕТВИ, ТЕЛА И СУСТАВНОГО ОТРОСТКА НИЖНЕЙ ЧЕЛЮСТИ
}

В челюстно-лицевой хирургии нередко встречаются дефекты нижней челюсти, включающие мыщелковый отросток. Особенно часто такие дефекты образуются после оперативных вмешательства по поду диффузного токсического остоенекроза нижней челюсти. Такие оперативные вмешательства приводят к образованию послеоперационных дефектов и сопровождаются глубокими морфологическими, функциональными и эстетическими нарушениями.

Результаты реконструктивного лечения влияют на восстановление функции речи и внешний вид пациента, что требует более высокой точности качества проведения хирургического вмешательства [1].

Использование цифровых технологии, таких как $3 d$ изображения на основе компьютерной томографии, виртуальное планирование оперативного вмешательства, и 3d печать, широко используются в современной медицине для повышения точности и качества диагностики и хирургического восстановления дефектов, нижней челюсти посредством эндопротезирования [2].

Использование современных компьютерных технологий позволяет провести точные биомеханические исследования, с целью моделирования конкретной клинической ситуации и выбора оптимального метода восстановления костного дефекта [3-5].

\section{ЦЕЛЬРАБОТЫ}

Анализ трехмерной конечно-элементной модели пострезекционного дефекта нижней челюсти в области тела, угла и мыщелкового отростка с целью имитации репозиции и фиксации фрагмента, проектирование и разработка индивидуального имплантата эндопротеза. Сравнение напряженно-деформационного состояния этой пластины со стандартной реконструктивной пластиной фирмы «Конмет», с точки зрения стабилизации сегментов нижней челюсти, путем моделирования жевательной нагрузки.

\section{МАТЕРИАЛ И МЕТОДЫ}

С целью изучения биомеханических свойств разработанной реконструктивной пластины по сравнению со стандартной реконструктивной пластиной фирмы «Конмет» была построена компьютерная модель нижней челюсти у пациентов с дефектом и дефектом тела, ветви и суставного отростка. На компьютерном томографе проведено исследование пациента, далее с использованием специального программного обеспечения «In Vesalius 3.01» на каждом компьютерном снимке выделяли костную ткань пациентов и отдельно от мягких тканей, которые соответствовали денситометрическим показателям. Для выделенных областей формировали контуры. После этого контуры, относящиеся к различным снимкам, составляли между собой с учетом заданной при томографии толщины слоя сканирования. Затем производили формирование модели в STL-формате в программе Mashmixer 4.0. На модели проводилось: выделение интересующей области, удаление артефактов, улучшение качества поверхностей, оптимизация полигональной сетки.

Компьютерное моделирование разработанной и стандартной реконструктивных пластины, фиксирующих винтов, а так же импорт твердотельные модели фрагмента нижней челюсти с последующим моделированием напряженнодеформационного состояния проводилось с использованием программного обеспечения ANSYS 19.1 (ANSYS Inc., Хьюстон, США)

( ) И.В. Чайковская, М.Ю. Павленко, С.Н. Царенко, 2020 (c) Университетская Клиника, 2020 
Таблица 1.

Физические свойства материалов, применяемые в исследовании

\begin{tabular}{lcccc}
\hline & $\begin{array}{c}\text { Наименование } \\
\text { материала }\end{array}$ & $\begin{array}{c}\text { Модуль Юнга } \\
(\mathrm{GPa})\end{array}$ & $\begin{array}{c}\text { Плотность } \\
\left(\mathrm{kг} / \mathrm{M}^{3}\right)\end{array}$ & $\begin{array}{c}\text { Коэффициент } \\
\text { пуассона }\end{array}$ \\
\hline \hline Нижняя челюсть & Кортикальная кость & 1,48 & 1850 & 0,28 \\
\hline $\begin{array}{l}\text { Индивидуальная } \\
\text { пластина }\end{array}$ & Титан марки ВТ 1-0 & 1,12 & 4505 & 0,342 \\
\hline $\begin{array}{l}\text { Стандартная пластина } \\
\text { «Конмет» }\end{array}$ & $\begin{array}{c}\text { Титан марки ASTM F67-00 } \\
\text { (Тінапіuт Gradium 2) }\end{array}$ & 1,05 & 4510 & 0,37 \\
\hline $\begin{array}{l}\text { Винты для фиксации } \\
\text { пластин }\end{array}$ & $\begin{array}{c}\text { Титан марки ASTM F-136 } \\
\text { (Ті 6Al-4V ELI) }\end{array}$ & 1,10 & 4430 & 0,31 \\
\hline
\end{tabular}

Параметры титановой реконструктивной пластины, эндопротеза суставного головки и винтов соответствовали данным, предоставленным производителем. Физические свойства материалов представлены в таблице (табл. 1.). Винты изготовлены из титанового сплава (титан-6 Aluminum-4 Vanadium; (ASTM F-136), a пластина-эндопротез «Конмет»- из технически чистого титана (ASTM F67).

Индивидуальная реконструктивная пластина для замещения дефекта нижней челюсти при отсутствии ветви тела и суставного отростка нижней челюсти [6] изготовлен из титана марки ВТ 1-0 (рис. 1.).

Она представляет собой криволинейную тонкостенную перфорированную, самонесущую оболочку 1 , которая в свою очередь имеет опорные элементы анатомически точно повторяющий поверхность концевого фрагмента 2, промежуточную часть, анатомически повторяющую отсутствующую часть нижней челюсти и мыщелковый отросток 3 , сквозные отверстия для фик- сации тканей к телу эндопротеза 4, сквозные отверстия для горизонтальной бикортикальной фиксации к костному дефекту 5 , сквозные отверстия для вертикальной кортикальной фиксации к костному дефекту 6, сквозные отверстия для фиксации височной мышцы 7.

В ходе эксперимента был проведен сравнительный анализ нижней челюсти с дефектом тела, ветви и мыщелкового отростка В сравнении участвовала стандартная реконструктивная пластина фирмы «Конмет», которая фиксировалась 4 винтами (рис. 2.), и разработанная индивидуальная реконструктивная пластина, которая также имела для фиксации 4 винта (рис. 3.).

Считалось, что каждая пластина и винт находятся в идеальном контакте с костью, при этом учитывалось трение. Коэффициенты трение составили: между титаном и костью 0,3 , между титаном и титаном 0,55 . Винты, соединяющие пластины с фрагментами нижней челюсти, были смоделированы с резьбой и пронумерованы.

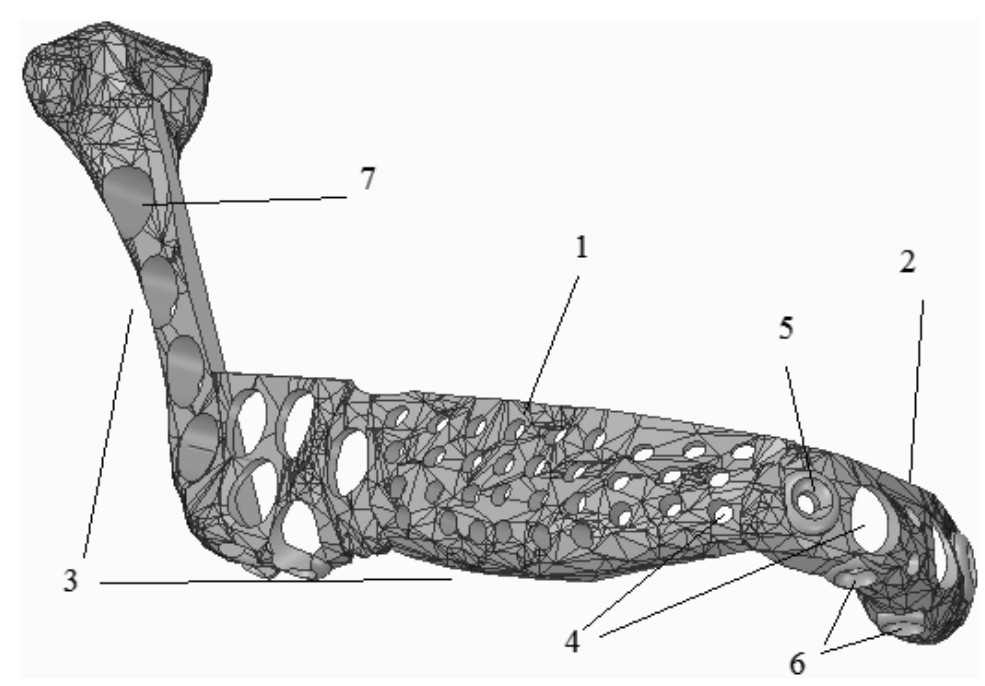

Рис. 1. Схема разработанной индивидуальной реконструктивной пластины. 1 - самонесущая оболочка, 2 концевой фрагмент, 3 - промежуточную часть, 4 -отверстия для фиксации тканей к телу эндопротеза, 5 - отверстия для горизонтальной бикортикальной фиксации к костному дефекту, 6 - отверстия для вертикальной кортикальной фиксации к костному дефекту, 7 - отверстия для фиксации височной мышцы. 


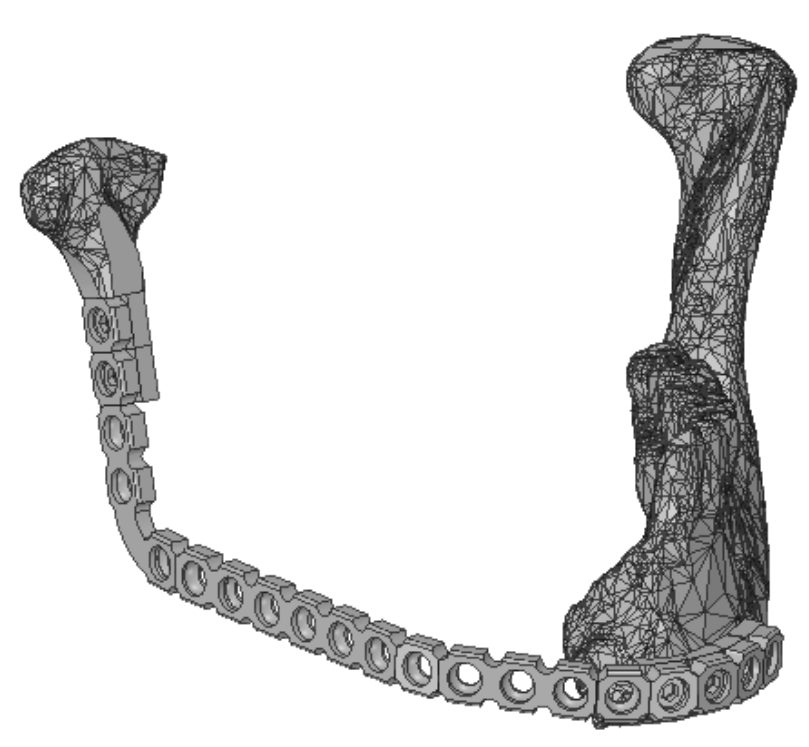

Рис. 2. Компьютерная модель стандартной реконструктивной пластины фирмы «Конмет» фиксированная 4 винтами к фрагменту нижней челюсти

Сборка конечных элементов, содержащая индивидуальную реконструктивную пластину четыре винта и фрагмент нижней челюсти, состояла из 174049 узлов и 98701 элементов.

Сборка конечных элементов, содержащая стандартную реконструктивную пластину четыре винта и фрагмент нижней челюсти, состояла из 130057 узлов и 73818 элементов. В обеих сборках в качестве объемного конечного элемента использовался тетраэдр.

С целью имитации биомеханических нагрузок, которые испытывают пластины при жевании была приложена сила при окклюзионном контакте резца. Величина вертикальной нагрузки принята равной 150 Н. Мышелковый отросток, как и на нижней челюсти, так и на пластинах был зафиксирован во всех трех направлениях для предотвращения силы реакции в височно-нижнечелюстном суставе.

В качестве критерия оценки приняты эквивалентные напряжения по фон Мизесу [6]. На основании принятого критерия считалось, что если максимальный напряжение на растяжение для каждой структуры (кости или винта) превышено, структура может выйти из строя.

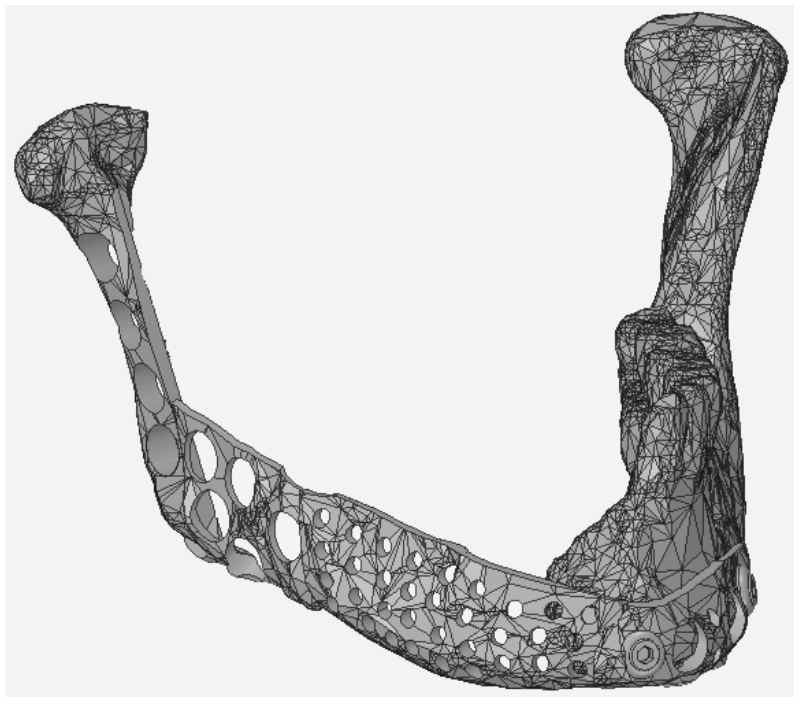

Рис. 3. Компьютерная модель индивидуальной реконструктивной пластины фиксированная 4 винтами к фрагменту нижней челюсти

\section{РЕЗУЛЬТАТЫ И ОБСУЖДЕНИЕ}

Изучение полного перемещения трехмерных конечно-элементной моделей показали, что при вертикальной нагрузке 150 Н в область клыка индивидуальная пластина лучше выдерживает осевые нагрузки. Согласно данным таблицы 2 индивидуальная пластина находится под меньшим напряжением, чем стандартная пластина. Напряжение в основном сосредоточено в области винтов в ментальном отделе большого фрагмента нижней челюсти.

В таблице 3 представлены значения эквивалентных напряжений по фон Мизису для каждой сборки (винты, пластина, фрагменты челюсти вместе), и для каждого винта по отдельности.

При сравнении двух методов фиксации, более высокое значение эквивалентных напряжений по фон Мизису в модели индивидуально реконструктивной пластине, чем в стандартной пластине. Такое значение объясняются большей биомеханической стабильностью разработанной пластины, которая обеспечивается более плотным прилеганием пластины к фрагментом костной ткани и как следствием большей пло-

Сравнение значений полного перемещения объектов

Таблица 2. для разработанной и стандартной реконструктивной пластины

\begin{tabular}{lcc}
\hline & $\begin{array}{c}\text { Разработанная реконструктивная } \\
\text { пластина (мм). }\end{array}$ & $\begin{array}{c}\text { Стандартная реконструктиная } \\
\text { пластина (мм). }\end{array}$ \\
\hline \hline Максимальное значение & 1,52 & 12,41 \\
\hline Среднее значение & 1,31 & 7,42 \\
\hline
\end{tabular}


Значения эквивалентных напряжений по фон Мизису

Таблица 3.

\begin{tabular}{lcc}
\hline Элементы & $\begin{array}{c}\text { Разработанная реконструктивная } \\
\text { пластина, критерий фон Мизиса (MPa) }\end{array}$ & $\begin{array}{c}\text { Стандартная реконструктиная пластина, } \\
\text { критерий фон Мизиса (MРа) }\end{array}$ \\
\hline \hline Вся структура & 245,26 & 181,27 \\
\hline 1 винт & 222.457 & 143,34 \\
\hline 2 винт & 251.29 & 142,45 \\
\hline 3 винт & 217.78 & 139,22 \\
\hline 4 винт & 227.56 & 166,52 \\
\hline
\end{tabular}

скости соприкосновения, а так же разными направлением фиксирующих винтов.

\section{З А К ЛЮ Ч Н ИЕ}

Таким образом, разработанная индивидуальная реконструктивная пластина для фиксации фрагмента нижней челюсти обладает бо- лее лучшими прочностными характеристиками, обеспечивает более жесткую фиксацию по сравнению со стандартной реконстркутивной пластиной фирмы «Конмет» и способствуют уменьшению развития наиболее частого осложнения - резорбции фиксирующих винтов и, как следствие, потери фиксации пластины.

\section{И.В. Чайковская ${ }^{1}$ М.Ю. Павленко, С.Н. Царенко 2 \\ ${ }^{1}$ ГОО ВПО «Донецкий национальный медицинский университет имени М. Горького», Донецк

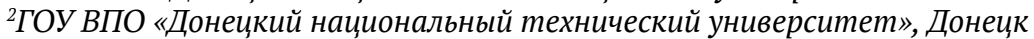 \\ КОНЕЧНО-ЭЛЕМЕНТАРНЫЙ АНАЛИЗ НАПРЯЖЕННО-ДЕФОРМИРОВАННОГО СОСТОЯНИЯ СТАНДАРТНОЙ И ИНДИВИДУАЛЬНОЙ РЕКОНСТРУКТИВНОЙ ПЛАСТИН ДЛЯ ЗАМЕЩЕНИЯ ДЕФЕКТА ВЕТВИ, ТЕЛА И СУСТАВНОГО ОТРОСТКА НИЖНЕЙ ЧЕЛЮСТИ}

Целью данного исследования был анализ трехмерной конечно-элементной модели пострезекционного дефекта ветви тела и суставного отростка нижней челюсти, проектирование и разработка индивидуальной реконструктивной пластины для замещения дефекта, сравнение напряженно-деформационного состояния этой пластины с угловой реконструктивной пластиной фирмы «Конмет» совмещенной с имплантатом суставного отростка, путем моделирования жевательной нагрузки.

Исследование было проведено на двух идентичных конечно-элементарных моделях костных дефектов нижней челюсти в которой фиксированы с угловой реконструктивной пластиной фирмы «Конмет» совмещенной с имплантатом суставного отростка, путем моделирования жевательной нагрузки, и раз- работанной реконструктивной пластиной. С целью имитации биомеханических нагрузок, которые испытывают пластины при жевании была приложена сила при окклюзионном контакте резца. Величина вертикальной нагрузки принята равной 150 Н. Мышелковый отросток зафиксирован во всех трех направлениях для предотвращения силы реакции в височнонижнечелюстном суставе.

В ходе эксперимента установлено что разработанная индивидуальная реконструктивная пластина обладает более лучшими прочностными характеристиками по сравнению со стандартной реконструктивной пластиной фирмы «Конмет».

Ключевые слова: конечно-элементный анализ, дефект ветви тела и суставного отростка нижней челюсти, реконструктивная пластина.

\section{M.U. Pavlenko', I.V. Chaykovskaya ${ }^{1}$, S.N. Tsarenko ${ }^{2}$}

${ }^{1}$ SEI HPE «M. Gorky Donetsk National Medical University», Donetsk

${ }^{2} S H E E$ «Donetsk National Technical University», Donetsk

\section{THE FINITE ELEMENTARY ANALYSIS OF THE STRESS-STRAIN STATE IS A STANDARD AND INDIVIDUAL RECONSTRUCTIVE PLATE FOR DEFECTS IN THE BRANCH, BODY AND ARTICULAR PROCESS OF THE LOWER JAW}

The purpose of the study was to analyze a three-dimensional finite element model of a post-resection defect in a body branch and an articular process of the low- er jaw, to design and develop an individual reconstructive plate to replace the defect, to compare the stress-strain state of this plate with the angular reconstructive plate of 
«CONMET» firm combined with an implant of the articular process through a simulation of the chewing load.

The study was carried out on two identical finite elementary models of bone defects of the lower jaw in which are fixed with an angular «CONMET» plate combined with an implant of the articular process, by modeling a chewing load, and designed reconstructive plate. In order to simulate the biomechanical loads on the plates when chewing was applied an impact of force during the occlusal contact of the incisor. The magnitude of the verti- cal load was equal to $150 \mathrm{H}$. The condylar process is fixed in all three directions to prevent reaction forces in the temporomandibular joint.

During the experiment, it was established, that the developed individual reconstructive plate has better strength characteristics compared to the standard reconstructive plate «CONMET».

Keywords: finite element analysis, defect in the branch of the body and articular process of the lower jaw, reconstructive plate.

\section{ЛИТЕРАТУРА}

1. Рудык А.Н., Бусыгин М.А., Хамидуллин Р.Г., Чернышев В.А., Хамитов М.Р., Жаворонков В.В., Минвалеев Р.И., Уткузов А.Р., Чернов М.С. Опыт применения технологий $3 \mathrm{~d}$ визуализации и принтинга в клинической практике при реконструкции дефектов нижней челюсти при злокачественных новообразованиях. Поволжский онкологический вестник. 2017; 4 (31): 25-29.

2. Диков Ю.Ю., Соболевский В.А., Кропотов М.А., Ивашков В.Ю. Применение трехмерного моделирования и 3D-печати при реконструкции нижней челюсти. Опухоли головы и шеи. 2015; 1: 22-26.

3. Atik F., Atac M.S., Özkan A., Kilinc Y., Arslan M. Biomechanical analysis of titanium fixation plates and screws in mandibular angle fractures. Nigerian Journal of Clinical Practice. 2016; 19 (3): 386-390. doi: 10.4103/11193077.179292

4. Goulart D.R. Kemmoku D.T., Noritomi P.Y., Márcio de Moraes Development of a Titanium Plate for Mandibular Angle Fractures with a Bone Defect in the Lower Border: Finite Element Analysis and Mechanical Test. J Oral Maxillofac 2015; 6 (3): e5. doi: 10.5037/jomr.2015.6305

5. Jomjunyong K., Rungsiyakull P., Rungsiyakull C., Aunmeungtong W., Chantaramungkorn M., Khongkhunthian P. Stress distribution of various designs of prostheses on short implants or standard implants in posterior maxilla: a three dimensional finite element analysis. ORAL \& Implantology. 2017; 10 (4): 369-380. doi: 10.11138/ orl/2017.10.4.369

6. Павленко М.Ю., Жданов В.Е., Гурин И.В. Патент Украины № 111143; 2015

\section{REFERENCES}

1. Rudyk A.N., Busygin M.A., Khamidullin R.G., Chernyshev V.A., Khamitov M.R., Zhavoronkov V.V., Minvaleev R.I., Utkuzov A.R., Chernov M.S. Opyt primeneniya tekhnologii $3 \mathrm{~d}$ vizualizatsii i printinga v klinicheskoi praktike pri rekonstruktsii defektov nizhnei chelyusti pri zlokachestvennykh novoobrazovaniyakh. Povolzhskii onkologicheskii vestnik. 2017; 4 (31): 25-29 (in Russian).

2. Dikov Yu.Yu., Sobolevskii V.A., Kropotov M.A., Ivashkov V.Yu. Primenenie trekhmernogo modelirovaniya i 3Dpechati pri rekonstruktsii nizhnei chelyusti. Opukholi golovy i shei. 2015; 1: 22-26 (in Russian).

3. Atik F., Atac M.S., Özkan A., Kilinc Y., Arslan M. Biomechanical analysis of titanium fixation plates and screws in mandibular angle fractures. Nigerian Journal of Clinical Practice. 2016; 19 (3): 386-390. doi: 10.4103/11193077.179292

4. Goulart D.R. Kemmoku D.T., Noritomi P.Y., Márcio de Moraes Development of a Titanium Plate for Mandibular Angle Fractures with a Bone Defect in the Lower Border: Finite Element Analysis and Mechanical Test. J Oral Maxillofac 2015; 6 (3): e5. doi: 10.5037/jomr.2015.6305

5. Jomjunyong K., Rungsiyakull P., Rungsiyakull C., Aunmeungtong W., Chantaramungkorn M., Khongkhunthian P. Stress distribution of various designs of prostheses on short implants or standard implants in posterior maxilla: a three dimensional finite element analysis. ORAL \& Implantology. 2017; 10 (4): 369-380. doi: 10.11138/ orl/2017.10.4.369

6. Pavlenko M.Yu., Zhdanov V.E., Gurin I.V. Patent Ukrainy № $111143 ; 2015$ 